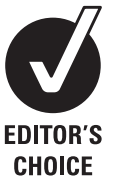

Institute of Naval Medicine Alverstoke, Hampshire, UK ${ }^{2}$ Academic Department of Military Emergency Medicine, Institute of Research and Development, Birmingham Research Park, Birmingham, UK ${ }^{3}$ The Royal Free Hospital, London, UK

${ }^{4}$ Academic Department of Military Surgery and Trauma, Institute of Research and Development, Birmingham Research Park, Birmingham, UK

Correspondence to Surg Lt Cdr D C W Henning, Institute of Naval Medicine, Crescent Road, Alverstoke, Hampshire P012 2DL, UK; danielhenning@mac.com

Copyright statement (C) British Crown Copyright 2009/MOD. Published with the permission of the Controller of Her Britannic Majesty's Stationery Office. This study was presented at the North Atlantic Treaty Organisation Medical Conference, Portugal, September 2009

Accepted 20 March 2010 Published Online First 20 July 2010

\title{
A comparison of civilian (National Confidential Enquiry into Patient Outcome and Death) trauma standards with current practice in a deployed field hospital in Afghanistan
}

\author{
D C W Henning, ${ }^{1}$ J E Smith, ${ }^{2}$ D Patch, ${ }^{3}$ A W Lambert ${ }^{4}$
}

\begin{abstract}
Background The National Confidential Enquiry into Patient Outcome and Death (NCEPOD) report on trauma management, published in 2007, defined standards for United Kingdom (UK) hospitals dealing with trauma. This study compared the NCEPOD standards with the performance of a UK military field hospital in Afghanistan. Setting UK military field hospital, Camp Bastion, Helmand Province, Afghanistan.

Materials and methods Data were collected prospectively for all patients fulfilling the trauma team activation criteria during the 3 months of Operation Herrick IXa (from mid October 2008 to mid January 2009) and combined with a retrospective review of prehospital documentation, trauma resuscitation notes, operations notes and transfer notes for these patients. Results During the study period, there were 226 trauma team activations. Of those patients brought to the medical facility at Camp Bastion by UK assets, 93.7\% were accompanied by a doctor with advanced airway skills, although only $6.2 \%$ of the patients required such an intervention. Consultants in emergency medicine and anaesthesia were present in $100 \%$ of cases and were directly involved (in either leading the team or performing airway management) in $63.5 \%$ and $77.6 \%$ of cases respectively. Of those patients requiring operative intervention, $98.1 \%$ had this performed by a consultant surgeon. Of those patients requiring CT, $93.6 \%$ of cases had this performed within $1 \mathrm{~h}$ of arrival.

Conclusions Trauma patients presenting to the medical facility at Camp Bastion during Operation Herrick IXa, irrespective of their nationality or background, received a high standard of medical care when compared with the NCEPOD standards.
\end{abstract}

The United Kingdom (UK) National Confidential Enquiry into Patient Outcome and Death (NCEPOD) report on trauma management was published in 2007 . $^{1}$ It found that almost $60 \%$ of patients studied received substandard care, with frequent deficiencies in organisational and clinical aspects of care. It defined standards of care for hospitals receiving trauma patients, summarised in box 1 .

The deployed UK military field hospital in Camp Bastion, Helmand Province, Afghanistan delivers care to injured coalition forces (International Stability Assistance Force) and Afghan security forces personnel and local civilians. It was established in 2006 in support of coalition operations in the Helmand province and is a purpose-built facility staffed by consultants in emergency medicine, general surgery, orthopaedic surgery, anaesthesia, intensive care and internal medicine. It has a 4 bedded resuscitation room, two operating tables, a laboratory, digital $x$ ray and CT, 4 intensive care beds and two general wards (at the time of this study). Prehospital retrieval of injured patients to the field hospital is usually achieved by a helicopter-borne medical team, termed the Medical Emergency Response Team (MERT). These are made up of a core team of a paramedic and an experienced critical care nurse and can be enhanced by the addition of a doctor, usually a specialist (either consultant or senior registrar) in emergency medicine or anaesthesia, at which time it is termed MERT-enhanced or MERT (E). At the time of this study, the MERT medical personnel were made up of four consultants and one senior registrar, who worked a one-in-five rota.

Benchmarking deployed trauma care against national standards is an important component of military trauma system quality assurance. Trauma governance is vital to ensure that deployed troops are treated appropriately and safely. ${ }^{2}$

The aim of this study was to compare trauma care in a forward military environment, delivered predominantly by the UK Defence Medical Services (DMS), with civilian standards as defined by NCEPOD.

\section{METHODS}

The Operation Herrick IXa took place in a 3-month period between mid October 2008 and mid January 2009. During this period, data were collected prospectively for all patients fulfilling the trauma team activation criteria as part of the routine collection of information for the Major Trauma Audit for Clinical Effectiveness ${ }^{2}{ }^{3}$ and combined with a subsequent retrospective review of prehospital documentation, operation notes and transfer notes for these patients.

All patients presenting to the field hospital emergency department (ED) who precipitated trauma team activation were included. The trauma team activation criteria are given in box 2 . A Microsoft Excel spreadsheet was used to collect and analyse the data. Each case was compared with NCEPOD standards.

\section{RESULTS}

During the study period, 226 patients fulfilled the inclusion criteria, which resulted in activation of the trauma team. Notes and documentation from seven patients (3.1\%) could not be accessed, and these patients were excluded from further analysis. Twenty-nine patients (13.2\%) were aged 16 years or younger (mean age 7.5 years), and the mean age of the 


\section{Pre-hospital care}

All agencies involved in trauma management, including emergency medical services, should be integrated into the clinical governance programmes of a regional trauma service. Airway management in trauma patients is often challenging, and the pre-hospital response for these patients should include someone with the skill to secure the airway, (including the use of rapid sequence intubation), and maintain adequate ventilation.

\section{Hospital reception}

A trauma team should be available $24 \mathrm{~h}$ a day, 7 days a week. This is an essential part of an organised trauma response system. A consultant must be the team leader for the management of the severely injured patient.

\section{Airway and breathing}

The current structure of prehospital management is insufficient. There is a high incidence of failed intubation and a high incidence of patients arriving at hospital with a partially or completely obstructed airway. Change is urgently required to provide a system that reliably provides a clear airway with good oxygenation and control of ventilation. This may be through the provision of personnel with the ability to provide anaesthesia and intubation in the prehospital phase or through the use of alternative airway devices.

\section{Circulation}

Trauma laparotomy is extremely challenging and requires consultant presence within the operating theatre. If CT is to be performed, all necessary images should be obtained at the same time, and routine use of head-to-toe scanning is recommended in the adult trauma patient if no indication for immediate intervention exists.

\section{Head injuries}

Patients with severe head injury should have a CT of the head performed as soon as possible after admission and within 1 hour of arrival at the hospital. All patients with severe head injury should be transferred to a neurosurgical critical care centre irrespective of the requirement for surgical intervention.

\section{Transfers}

There should be standardised transfer documentation of patient details, injuries, results of investigations and management, with records kept at the dispatching and receiving hospitals.

190 adult patients was 27.0 years. One hundred and thirty-nine patients (63.5\%) were Afghan (civilian, Afghan National Army or Afghan National Police), and 78 patients (35.6\%) were coalition forces (UK, US or Danish). There were 204 (93.2\%) male and 15 female patients. One hundred and ninety patients (86.8\%) were classed as battle injured, with injuries resulting from enemy action.

The most common mode of prehospital transport was MERT (E), the helicopter-borne physician-paramedic MERT. One hundred and ninety-four patients (88.6\%) were brought to Camp Bastion by MERT(E). The MERT (without physician) transported 13 patients (5.9\%), and the US Forces (paramedic only) transported 12 (5.5\%). Twelve patients (5.5\%) had an advanced airway intervention performed before arrival in the ED, of whom two had surgical airways performed by either medical officers or medics (combat medical technicians or medical assistants) before the arrival of the MERT; the remainder were endotracheal intubations performed by the MERT physician during rapid sequence induction of anaesthesia.

The presence of a consultant as either a team leader in the ED or a direct supervisor of the specialist registrar leading was $100 \%$.

With regard to airway management, during the study period, the physician on the MERTwas either a consultant or a specialist registrar in anaesthesia delivering prehospital medical care, and the same was true of the airway physician on the trauma team. Airway management was either performed or directly supervised by a consultant grade anaesthetist in $100 \%$ of cases.

One hundred and sixty-one patients $(73.5 \%)$ proceeded to the operating theatre, and $58(26.5 \%)$ did not require surgery. Surgical care was largely consultant delivered and led: consultant input was recorded if the name of a consultant was present on the operating note, either as the primary or assisting surgeon. According to these criteria, three cases (1.9\% of those requiring surgery) were performed solely by a non-consultant grade surgeon, all of which were minor procedures such as superficial wound debridement.
CT was performed in 78 cases $(35.6 \%)$ as part of the initial management. Our standard is to transfer the patient to the CT scanner within 60 min of arrival in the ED should it be required, and this was met in 73 cases (93.6\%), with a mean time to CT of $42 \mathrm{~min}$ and a median of $37 \mathrm{~min}$.

For documentation, the ED record of all trauma patients was in the form of the DMS Trauma Chart. Of patients discharged from the Camp Bastion facility, 100\% had appropriate transfer documentation.

\section{DISCUSSION}

Medical care for military personnel on overseas operations has been the subject of intense media and public scrutiny. In fact, data suggest several cases of unexpected survival in trauma patients on deployed operations. ${ }^{2}$

In the NCEPOD survey of UK practice, organisation of prehospital care, trauma team response, involvement of senior medical staff and immediate in-hospital care was found to be deficient in many hospitals. Public awareness of these deficiencies was cited in the report as being potentially useful in any debate about configuration of trauma services, and similarly the public need to have an accurate account of medical care given to deployed troops. Severe trauma is uncommon in the UK, and many hospitals see less than one severely injured patient per week, compared with a mean of 18 trauma team activations in the Camp Bastion facility per week during the study period.

Most $(88.6 \%)$ trauma patients in this study were treated in the prehospital phase and were transported to Camp Bastion by an experienced doctor skilled in advanced airway management. This is in line with current military doctrine but is not a routine practice in the UK. These practitioners worked in the field hospital when not on MERT duties and took part in the internal education and clinical governance programme. Prehospital clinical records are matched with the in-hospital documentation and 


\section{Box 2 Trauma team activation criteria ${ }^{3}$}

\author{
Mechanism/history \\ Penetrating trauma \\ - Gunshot or shrapnel wound \\ - Blast injury (mine/improvised explosive devices/grenade) \\ - Stab wound \\ Blunt trauma \\ - Motor vehicle crash with ejection \\ - Motorcyclist or pedestrian hit by vehicle at a speed of \\ $>30 \mathrm{~km} / \mathrm{h}$ \\ - Fall $>5 \mathrm{~m}$ \\ - Death in the same vehicle \\ - Entrapment and/or crush injury \\ - Interhospital trauma transfer meeting activation criteria \\ and \\ Anatomy \\ - Injury to two or more body regions \\ - Fracture to two or more long bones \\ - Spinal cord injury \\ - Amputation of a limb \\ - Penetrating injury to the head, the neck, the torso or the \\ proximal limb \\ - Burns $>15 \%$ of the body surface area in adults or $>10 \%$ in \\ children, or airway burns \\ - Airway obstruction \\ or \\ Physiology \\ - Systolic blood pressure $<90 \mathrm{~mm} \mathrm{Hg}$ or pulse $>120$ beats per \\ minute (adults) \\ - Respiratory rate $<10$ or $>30$ (adults); $\mathrm{SaO}_{2}<90 \%$ \\ - Depressed level of consciousness, or fitting \\ - Deterioration in the ED \\ - Age $>70$ years \\ - Pregnancy $>24$ weeks with torso injury
}

audited centrally by the Academic Department of Military Emergency Medicine, thus satisfying a combined audit and governance umbrella covering prehospital and in-hospital care.

The availability of a consultant as trauma team leader in $100 \%$ of cases meets NCEPOD recommendations and is essential for the optimal outcome of these complex patients. The $100 \%$ availability of a consultant in anaesthesia to provide airway management is similarly important. In single-casualty presentations, the team leader was always either the consultant or the registrar in emergency medicine: in cases where the registrar led the team, the consultant was immediately available and was directly supervising. In multiple-casualty situations, consultants in general and orthopaedic surgery, anaesthesia and intensive care were invited to lead the trauma teams.

The pattern of injuries sustained during Herrick IXa was complex, and this was reflected in the almost universal (98.1\%) presence of a consultant surgeon during operative intervention. In addition to this, the same consultants were present during the initial resuscitation phase, and this was vital when prioritising multiple casualties for theatre.

In five patients, CT was not performed within $60 \mathrm{~min}$ of arrival in the ED. Further analysis of these cases revealed that they all occurred during multiple serious-casualty situations where several patients presenting simultaneously required imaging and, therefore, had to be prioritised. Pending CT, patients were either managed in the $\mathrm{ED}$ or the intensive care unit depending on available resources.

One of the NCEPOD standards is the transfer of all neurotrauma patients to a neurosurgical centre, irrespective of the need for operative intervention. This is one area that the Camp Bastion facility was deficient, in that there were no specialist neurosurgical facilities in the Helmand province. The neurosurgical intensive care unit in Kandahar (the nearest such unit, approximately 30 min flight time) could only accommodate four patients at one time. Head-injured patients who did not require neurosurgical intervention were, therefore, managed in the intensive care unit in Camp Bastion, if discussion with a neurosurgeon deemed this appropriate. In military doctrine, evacuation of an intracranial haematoma may be performed by a general or orthopaedic surgeon, depending on the access to specialist facilities.

UK DMS assets use uniform prehospital, trauma resuscitation, anaesthetic and operative documentation. Patient documentation on transfer from Camp Bastion depended on which centre the patient was being transferred to. Patients being transferred to the medical facility at Kandahar or repatriated to the UK had photocopies of all notes, images and scans in addition to a transfer summary.

The UK DMS closely audits all trauma data. A dedicated trauma nurse coordinator, usually an experienced emergency nurse, is part of the trauma team. The trauma nurse coordinator acts either as the trauma chart scribe or as a supervisor of the scribe during the resuscitation and has no direct clinical role. Data continue to be collected as the patient proceeds through radiology, surgery and ward care (as appropriate) in addition to when they are evacuated back to the UK and discharged to outpatient follow-up. These data are analysed at the Academic Department of Military Emergency Medicine and are used to shape medical organisation and provide insight into equipment issues (eg, the effectiveness of body armour).

Since the completion of this study, the Healthcare Commission have published a report on the care provided by the DMS to its frontline troops and described trauma management on overseas operations as exemplary and exceptional. ${ }^{4}$ Calls for cooperation between the DMS and the National Health Service to improve trauma care for all patients have been welcomed by clinicians in both organisations.

\section{CONCLUSION}

This study demonstrates that a deployed UK field hospital can deliver a high standard of care that, in most areas, equals or surpasses the recommendations for good civilian trauma care described in the 2007 NCEPOD trauma report. ${ }^{1}$ A key feature of these recommendations is the input of senior clinical decision makers early in the management of complex trauma patients.

Acknowledgements The authors thank all those involved in the care of trauma patients presenting to the Camp Bastion field hospital during Operation Herrick IXa

Competing interests All authors are serving members of HM Armed Forces.

Provenance and peer review Not commissioned; externally peer reviewed

\section{REFERENCES}

1. NCEPOD. Trauma: who cares? London: National Confidential Enquiry into Patient Outcome and Death, 2007.

2. Hodgetts TJ, Davies S, Midwinter M, et al. Operational mortality of UK service personnel in Iraq and Afghanistan: a one-year analysis 2006-7. J R Army Med Corps 2007:153:252-4.

3. Joint Doctrine Publication 4-03.1. Clinical guidelines for operations. London: Defence Medical Services Department, 2008.

4. Healthcare Commission. A review of the clinical governance of the Defence Medical Services in the UK and overseas. London: Defence Medical Services, 2009. 